\title{
Avaliação da função visual em pacientes com distrofia de cones
}

Visual function assessment in patients with cone dystrophy

Marta Sato

Adriana Berezovsky

Paula Yuri Sacai ${ }^{2}$

Solange Rios Salomão3

\section{RE S UMO}

Objetivo: Avaliar a função visual pela eletrorretinografia de campo total e pela acuidade visual em pacientes com distrofia de cones. Métodos: Um grupo de 23 pacientes ( 16 do sexo feminino e 7 do sexo masculino) com distrofia de cones foi avaliado no Laboratório de Eletrofisiologia Visual Clínica do Departamento de Oftalmologia da Universidade Federal de São Paulo. A avaliação constou de: sinais e sintomas, acuidade visual medida pela tabelaETDRS ou de Snellen e função retiniana pela eletrorretinografia de campo total. As amplitudes pico-a-pico $(\mu \mathrm{V})$ e o tempo de culminação da onda- $b(\mathrm{~ms})$ foram comparadas com normas descritas na literatura. Resultados: A acuidade visual no melhor olho variou de 0,07 a 3,00 logMAR (20/25-MM a $10 \mathrm{~cm})$, com média de 0,85 $\pm 0,64 \log$ MAR $(20 / 140)$. A média da amplitude pico-a-pico das respostas escotópicas de bastonetes foi de $144,29 \pm 80,05 \mu \mathrm{V}$ com média do tempo de culminação da onda- $b$ de $92,85 \pm 9,02 \mathrm{~ms}$ (normal para aidade). A média da amplitude pico-a-pico para respostas fotópicas de cones foi de $5,06 \pm 8,59 \mu \mathrm{V}$ (reduzida para a idade) com média do tempo de culminação da onda- $b$ de $43,4 \pm 14,5$ ms (atrasado para a idade) nos pacientes com resposta detectável e para as respostas do flicker, a amplitude foi de 5,00 $\pm 9,00 \mu \mathrm{V}$ (reduzida para aidade) com média do tempo de culminação da onda- $b$ de $32,90 \pm 12,87$ ms (atrasado para a idade). Conclusão: A acuidade visual média encontrada neste grupo de pacientes com distrofia de cones foi de $0,85 \log \operatorname{MAR}(20 / 140)$. A fotofobia foi o sintoma mais freqüente juntamente com a baixa de visão e deficiência de visão de cores. Em 10/23 pacientes o diagnóstico foi feito pela eletrorretinografia, devido à ausência de alterações fundoscópicas.

Descritores:Eletromretinografia;Cones (retina)/fisiologia;Percepçãodecores;Acuidade visual;Baixavisão

I N T R O D U Ç ̃̃ O

O termo distrofia de cones é utilizado para achados morfológicos e funcionais normais em recém-nascidos, que progressivamente tornam-se alterados com o desenvolvimento de sintomas durante a vida ${ }^{(1)}$. Inicialmente as queixas são diminuição da acuidade visual, alteração nos contrastes e na visão de cores. Nistagmo e fotofobia também podem fazer parte do quadro clínico. As alterações oftalmológicas e funcionais freqüentemente precedem as mudanças fundoscópicas ${ }^{(2)}$. A distrofia de cones pode ser classificada em forma estacionária (acromatopsia) ou progressiva (doença progressiva de cones) $)^{(3-4)}$, podendo afetar os pigmentos da visão de $\operatorname{cores}^{(1,5)}$, acarretar em mudanças na acuidade visual ${ }^{(4)}$ e apresentar alterações fundoscópicas ${ }^{(3-4)}$. 
O objetivo deste estudo foi determinar a função visual de pacientes com distrofia de cones, pela medida da acuidade visual e pela eletrorretinografia de campo total, relacionandoos com os aspectos clínicos.

MÉ TODOS

\section{Participantes}

Foram estudados 23 casos de distrofia de cones no Laboratório de Eletrofisiologia Visual Clínica do Departamento de Oftalmologia da UNIFESP. A idade no dia da avaliação variou de 6 meses a 55 anos $(22,42 \pm 16,03$ anos). Sete eram do sexo masculino e 16 do sexo feminino.

\section{Procedimentos}

Os procedimentos empregados foram anamnese, medida de acuidade visual monocular com a melhor correção óptica possível pelas tabelas ETDRS ou Snellen, convertendo-se o valor obtido para unidade de medida $\log$ MAR. Os exames oftalmológicos consistiram de exame de refração, campo visual, pressão intra-ocular, biomicroscopia, fundoscopia e senso cromático quando possível. Os procedimentos éticos para a realização desta pesquisa foram rigorosamente obedecidos e o estudo obedeceu às normas da declaração de Helsinque.

\section{Eletrorretinografia de campo total}

O eletrorretinograma de campo total (ERG) foi realizado seguindo o protocolo padrão de ERG da International Society for Clinical Electrophysiology of Vision (ISCEV) ${ }^{(6)}$, após 30 minutos de adaptação ao escuro e dilatação máxima da pupila (diâmetro pupilar mínimo de $6 \mathrm{~mm}$ ), com uma gota de tropicamida a $1 \%$ e uma gota de fenilefrina a $10 \%$. Após a adaptação ao escuro, foi instilada uma gota de colírio de tetracaína (anestésico) e um eletrodo de lente de contato bipolar com filamento de ouro (GoldLens electrode, Doran Instruments, MD, USA) foi inserido no olho, com colírio de metilcelulose a $2 \%$ para proteção da superfície da córnea. Um eletrodo terra de cúpula de ouro em forma de clipe foi colocado no lobo da orelha ipsilateral, preenchido com gel eletrolítico. Para a realização do ERG foi utilizado o sistema eletrodiagnóstico UTAS E-3000 (LKC Technologies Inc., Gaithersburg, MD, USA) com amplificação de 10.000 vezes, com filtros eletrônicos variando de $0,3 \mathrm{~Hz}$ a $500 \mathrm{~Hz}$. As amplitudes da resposta foram medidas em microvolts $(\mu \mathrm{V})$ e o tempo de culminação em milisegundos (ms).

Em cada uma das cinco respostas do protocolo padrão da ISCEV, foram feitas 20 apresentações dos estímulos luminosos, sendo considerada a resposta final a média destes 20 registros, com exceção da resposta de flicker em que foi feita a média de 50 apresentações dos estímulos luminosos. Os registros foram obtidos na seguinte ordem:

1) Resposta escotópica de bastonetes, com estimulação de uma luz branca com intensidade máxima atenuada em 2,5 unidades logarítmicas por filtro de densidade neutra $\left(0,0098 \mathrm{~cd} / \mathrm{m}^{2}\right)$.
O intervalo interestímulos foi de 2 segundos. Este estímulo fica abaixo do limiar de resposta dos cones produzindo apenas respostas de bastonetes.

2) Resposta escotópica máxima, obtida com estímulos de alta intensidade $\left(2,467 \mathrm{~cd} / \mathrm{m}^{2}\right)$. Para esta resposta houve contribuição dos cones e bastonetes. O estímulo é apresentado ainda com o olho adaptado ao escuro e sem a presença de luz de fundo. O intervalo interestímulos foi de 10 segundos, devido à alta intensidade do mesmo.

3) Resposta escotópica de potenciais oscilatórios, com intervalo interestímulos de 1 segundo e filtro passa-alto de $75 \mathrm{~Hz}$. Os potenciais oscilatórios apresentam dois picos proeminentes aparecendo na porção ascendente da resposta escotópica máxima e refletem a atividade das camadas internas da retina.

4) Resposta fotópica de cones ao flash único, com intervalo interestímulos de 1 segundo, após pré-adaptação por $10 \mathrm{mi}-$ nutos a uma luz de fundo branca com luminância de $30 \mathrm{~cd} / \mathrm{m}^{2}$. Esta resposta contém uma onda- $a$ proeminente com tempo de culminação de aproximadamente $15 \mathrm{~ms}$, uma onda- $b$ com tempo de culminação de aproximadamente $30 \mathrm{~ms}$ e pelo menos duas oscilações logo depois da onda- $b$.

5) Resposta fotópica de flicker a $30 \mathrm{~Hz}$, resposta isolada dos cones com estimulação intermitente a uma freqüência de $30 \mathrm{~Hz}$, registrada na presença de uma luz de fundo com luminância de $30 \mathrm{~cd} / \mathrm{m}^{2}$, após um período de adaptação do olho à luz. A amplitude da resposta foi medida pico-a-pico, ou seja, o tamanho da resposta foi medido do pico da resposta mínima ao pico da resposta máxima. $\mathrm{O}$ tempo culminação foi medido a partir do início do estímulo até o pico máximo da onda- $b$.

A descrição dos pacientes é mostrada na Tabela I.

\section{RESULTADOS}

Os resultados dos ERGs foram comparados com as normas previamente descritas ${ }^{(7)}$.

Nenhum paciente apresentou história de consangüinidade na família. Do total dos pacientes analisados $13(56,5 \%)$ relataram algum membro familiar com a mesma patologia, sendo 3 irmãs de uma mesma família (pacientes 1, 2 e 14).

A acuidade visual no melhor olho variou de 0,07 a 3 logMAR (20/25-MM a 10cm), com média de $0,85 \pm 0,64 \log$ MAR (20/140). Vinte pacientes (87\%) apresentaram alteração na visão de cores; $19(82,6 \%)$ relataram visão noturna normal e/ou melhor, e $18(78,2 \%)$ pacientes queixaram-se de fotofobia importante à luz solar e ambientes muito claros. $\mathrm{O}$ nistagmo manifesto foi observado em 14 (60,9\%) pacientes; sendo 13 $(92,8 \%)$ predominantemente horizontal de alta frequiência e baixa amplitude e $1(7,2 \%)$ horizonto-rotatório (Tabela I).

Os exames fundoscópicos mostraram-se normais em 10 pacientes $(43,5 \%) ; 5(21,7 \%)$ apresentaram alterações maculares; 3 $(13,1 \%)$ com discreta alteração pigmentária difusa e 1 (4,3\%) com papilas pequenas e discretamente pálidas. Não foi possível o acesso ao resultado fundoscópico de 4 pacientes vindos de outros serviços. 


\begin{tabular}{|c|c|c|c|c|c|c|c|c|c|c|}
\hline PC & $\begin{array}{l}\text { Idade } \\
\text { (anos) }\end{array}$ & Sexo & HF & Nistagmo & Fotofobla & $\begin{array}{c}\text { AV(OD) } \\
\text { LogMAR }\end{array}$ & $\begin{array}{c}\text { AV(OE) } \\
\text { LOgMAR }\end{array}$ & $\begin{array}{l}\text { Visão } \\
\text { Notuma }\end{array}$ & $\begin{array}{l}\text { Visãode } \\
\text { Cores }\end{array}$ & FO \\
\hline 1 & 9,78 & $\mathbf{F}$ & Sim & Sim & Sim & 0,1 & 0,1 & nd & at & d \\
\hline 2 & 7,16 & $\mathbf{F}$ & Sim & Sim & Sim & 0,9 & 1 & n & atk & n \\
\hline 3 & 39,45 & M & Não & Não & Sim & 1 & 1 & n & at & maculopatia \\
\hline 4 & 8,88 & M & Sim & Sim & Sim & 0,9 & 0,9 & n & at & n \\
\hline 5 & 42,47 & $\mathbf{F}$ & Não & Sim & Sim & 1,3 & 1,2 & n & Alt(vd) & papilas peq. \\
\hline 6 & 25,60 & $\mathbf{F}$ & Não & Sim & Sim & 0,07 & 0,07 & n & at & altEPR \\
\hline 7 & 6,78 & $\mathbf{F}$ & Não & Sim & Sim & 1 & 0,8 & n & at & n \\
\hline 8 & 31,47 & $\mathbf{F}$ & Não & Sim & Não & 3 & 3 & piora & at & altEPR \\
\hline 9 & 54,75 & $\mathbf{F}$ & Não & Não & Sim & 1 & 1 & n & at & NR \\
\hline 10 & 16,78 & M & Não & Não & Sim & 1,3 & 1,3 & piora & n & maculopatia \\
\hline 11 & 7,46 & $\mathbf{F}$ & Não & Sin & Sim & 0,1 & 0,2 & n & at & n \\
\hline 12 & 34,61 & M & Não & Não & Não & 0 & 0 & n & n & NR \\
\hline 13 & 12,23 & $\mathbf{F}$ & Sim & Sin & Sim & 1 & 0,9 & n & at & NR \\
\hline 14 & 0,36 & $\mathbf{F}$ & Sim & Sim & Não & NR & NR & NR & NR & n \\
\hline 15 & 14,25 & $\mathbf{F}$ & Não & Não & Não & 2 & 1,2 & piora & at & maculopatia \\
\hline 16 & 13,92 & $\mathbf{F}$ & Sim & Sim & Sim & 1 & 0,9 & n & at & n \\
\hline 17 & 22,92 & M & Sim & Não & Sim & 0,8 & 0,9 & n & at & maculopatia \\
\hline 18 & 16,08 & $\mathbf{F}$ & Sim & Não & Sim & 1 & 0,9 & n & at & altdiffusa \\
\hline 19 & $\mathbf{4 , 2 8}$ & $\mathbf{F}$ & Sim & Sim & Sim & 0,85 & 0,85 & n & at & n \\
\hline 20 & 5,59 & M & Sim & Sim & Não & 0,9 & 0,9 & n & at & NR \\
\hline 21 & 31,30 & $\mathbf{F}$ & Sim & Sim & Sim & NR & NR & n & at & nd \\
\hline 22 & 44,50 & $\mathbf{F}$ & Sim & Não & Sim & 1 & 1 & n & at & n \\
\hline $\mathbf{2 3}$ & 44,67 & M & Sim & Não & Sim & 1 & 1 & n & at & maculopatia \\
\hline
\end{tabular}

Anteriormente aos resultados do EPR, as hipóteses diagnósticas iniciais foram de distrofia de cones em $6(26,1 \%)$ pacientes. $17(73,9 \%)$ tiveram outras hipóteses, dentre elas: 10 pacientes com nistagmo congênito; $3 \mathrm{com}$ altas miopias; 2 com doença de Stargardt; 1 com retinose pigmentária e 1 com lesões retro-quiasmáticas.

Os achados eletrorretinográficos são mostrados na tabela II e foram comparados com normas da literatura ${ }^{(7)}$. A média da amplitude pico-a-pico das respostas escotópicas de bastonetes foi de $144,29 \pm 80,05 \mu \mathrm{V}$ (normal para a idade) com média do tempo de culminação da onda- $b$ de $92,85 \pm 9,02 \mathrm{~ms}$ (normal para a idade). A média de amplitude pico-a-pico das respostas máximas escotópicas de bastonetes foi de $206,26 \pm 76,92 \mu \mathrm{V}$ (normal para a idade). A média de amplitude pico-a-pico das respostas do potencial oscilatório foi de $51,96 \pm 32,31 \mu \mathrm{V}$ (reduzido para a idade). A média de amplitude pico-a-pico das respostas de cones foi de $5,1 \pm 8,6 \mu \mathrm{V}$ (reduzido para a idade) com a média do tempo de culminação da onda- $b$ de $43,4 \pm 23,4 \mathrm{~ms}$ (atrasado para a idade) nos pacientes com resposta detectável. A média de amplitude pico-a-pico das respostas do flicker foi de $5 \pm 9 \mu \mathrm{V}$ (reduzido para a idade) com a média do tempo de culminação da onda- $b$ de $34,4 \pm 10,9 \mathrm{~ms}$ (atrasado para a idade).

D I S C US S ÃO

A distrofia de cones é uma doença retiniana hereditária que primariamente afeta a função do sistema de cones, com preservação ou envolvimento limitado do sistema de bastonetes, que surge com o avanço da doença ${ }^{(8)}$. A função visual nos pacientes com distrofia de cones é mais debilitada sob condições de adaptação à luz, e as perdas incluem redução na acuidade visual, fotofobia, sensibilidade ao ofuscamento (glare), e defeitos na visão de $\operatorname{cores}^{(3,5,8)}$.

A redução na acuidade visual foi um achado freqüente no grupo estudado, com apenas 4 pacientes (pacientes no $1,6,11$ e 12) apresentando acuidade melhor do que 20/30 no melhor olho. Esta variabilidade pode estar correlacionada com a idade por ocasião do início dos sintomas, sendo os casos mais recentes, os menos afetados pela redução na visão. No entanto, há relato de $\mathrm{AV}$ muito boa nos casos iniciais de distrofia progressiva de cones ${ }^{(9)}$. As queixas mais freqüentes nestes pacientes foram alteração na visão de cores, melhora da visão em ambientes escuros e fotofobia.

As mudanças fundoscópicas na distrofia de cones, tipicamente, são mínimas e limitadas à área macular, mas o fundus pode também parecer normal ${ }^{(10)}$. Foi detectada maculopatia em apenas 5 dos 23 pacientes estudados, com 10 apresentando ausência de alterações fundoscópicas.

O nistagmo pode estar presente e é mais comum nos casos de distrofia de cones congênita (acromatopsia). No grupo estudado, $61 \%$ dos pacientes apresentavam nistagmo. Um aspecto interessante a ser ressaltado é a ocorrência de nistagmo de pequena amplitude e alta frequiência, mesmo em pacientes 


\begin{tabular}{|c|c|c|c|c|c|c|c|}
\hline \multicolumn{8}{|c|}{ Tabela Il: Parâmetros eletrorretinográficos dos pacientes com distrofia de cones } \\
\hline $\begin{array}{l}\text { Paciente } \\
\text { n? }\end{array}$ & $\begin{array}{l}\text { Bastonetes } \\
\text { Amplitude } \\
(\mu \mathrm{V})\end{array}$ & $\begin{array}{c}\text { Bastonetes } \\
\text { Tempode } \\
\text { culminação } \\
\text { (ms) }\end{array}$ & $\begin{array}{c}\text { Máxima } \\
\text { resposta } \\
\text { Amplitude } \\
(\mu V)\end{array}$ & $\begin{array}{c}\text { Flicker } \\
\text { Amplitude } \\
(\mu \mathrm{V})\end{array}$ & $\begin{array}{l}\text { Flicker } \\
\text { Tempode } \\
\text { culminação } \\
\text { (ms) }\end{array}$ & $\begin{array}{c}\text { Cones } \\
\text { Amplitude } \\
(\mu \mathrm{V})\end{array}$ & $\begin{array}{c}\text { Cones } \\
\text { Tempode } \\
\text { culminação } \\
\text { (ms) }\end{array}$ \\
\hline 1 & 284,93 & 85 & 260,53 & 2 & 22,1 & 0 & 0 \\
\hline 2 & 292,86 & 87 & 273,04 & 2,2 & 21,4 & 0 & 0 \\
\hline 3 & 79,12 & $\boldsymbol{\infty}$ & 113,77 & 20,5 & 29,7 & 18,09 & 32 \\
\hline 4 & 157,41 & 99,5 & 143,52 & 2,7 & 33,6 & 0 & 0 \\
\hline 5 & 61,57 & 79,5 & 123,34 & 1,19 & 47,8 & 0 & 0 \\
\hline 6 & 86,68 & 87 & 237,3 & 4,73 & 18,2 & 21,28 & 75,5 \\
\hline 7 & 271,18 & 107 & 303,78 & 1 & 43,3 & 14,31 & 46,5 \\
\hline 8 & 92,19 & 86,5 & 134,77 & 35,7 & 32,9 & 31,38 & 33,5 \\
\hline 9 & 121,19 & 96,5 & 168,04 & 0,53 & 33 & 0 & 0 \\
\hline 10 & 80,8 & 91,5 & 160,4 & 0,77 & 38,2 & 6,4 & 34,5 \\
\hline 11 & 121,3 & 90,5 & 143,4 & 3,8 & 41,7 & 1,2 & 46,5 \\
\hline 12 & 105,3 & 81 & 154 & 22,7 & 30,6 & 10,7 & 31 \\
\hline 13 & 248,38 & 87,5 & 249,89 & 0,82 & 18,4 & 0 & 0 \\
\hline 14 & 94,48 & 95,5 & 118,99 & 6,5 & 48,3 & 7,46 & 55 \\
\hline 15 & 67,6 & 117 & 204,8 & 0,8 & 47,3 & 0 & 0 \\
\hline 16 & 117,1 & 87,5 & 303,7 & $\mathbf{4 , 4}$ & 31,4 & 0 & 0 \\
\hline 17 & 98,1 & 87 & 157,3 & 0,2 & 23,9 & 0 & 0 \\
\hline 18 & 109,4 & 97,5 & 152,7 & 0,6 & 44,8 & 0 & 0 \\
\hline 19 & 243,1 & 89 & 405,2 & 2,3 & 49,5 & 0 & 0 \\
\hline 20 & 206,2 & 98,5 & 291,1 & 0 & 0 & 0 & 0 \\
\hline 27 & 182,3 & 88 & 222,2 & 0,9 & 17,1 & 0 & 0 \\
\hline 22 & 90,4 & 103 & 155,4 & 1 & 48,1 & 0 & 0 \\
\hline$\overline{23}$ & 53,2 & 105 & 266,9 & 6,8 & 35,8 & 5,8 & 33,5 \\
\hline
\end{tabular}

com acuidade visual relativamente preservada (pacientes $\mathrm{n} 01$, 6 e 11). Muitas vezes este tipo de nistagmo pode passar desapercebido, tal a pequena extensão dos movimentos oscilatórios dos olhos.

Na distrofia de cones, o eletrorretinograma de campo total mediado pelos cones está reduzido em amplitude e atrasado. As respostas fotópicas isoladas de cones e do flicker a $30 \mathrm{~Hz}$ estão reduzidas ou até mesmo ausentes ${ }^{(4,10-11)}$. Os resultados apresentados neste estudo mostraram que houve uma redução drástica na amplitude pico-a-pico das respostas isoladas de cones, acompanhada de um prolongamento no tempo de culminação da onda- $b$ nos 9 casos em que foi possível registrar alguma resposta do sistema de cones. $\mathrm{O}$ mesmo padrão de resposta ocorreu à estimulação do flicker $30 \mathrm{~Hz}$. As alterações eletrorretinográficas fotópicas como a redução da amplitude pico-a-pico e o prolongamento de tempo de culminação da onda- $b$ estão concordantes com os resultados previamente relatados na literatura ${ }^{(2,4,11)}$.

Neste estudo, a fotofobia, a baixa acuidade visual e o defeito na visão de cores foram as alterações clínicas mais freqüentemente encontradas. Em 100\% dos pacientes deste estudo, os ERGs mostraram amplitudes fotópicas de cones e de flicker severamente reduzidas e atrasadas (média de $5 \mu \mathrm{V}$ e $43,4 \mathrm{~ms}$ ), confirmando o defeito difuso no sistema de cones. Os dados clínicos e eletrorretinográficos encontrados estão compatíveis com os da literatura ${ }^{(3-4,6,11)}$.

\section{O N C L U S Ã O}

Como os sintomas e sinais clínicos muitas vezes não são explicados pelo aspecto de fundo de olho, e pelo fato de que as alterações funcionais no ERG freqüentemente precedem as alterações fundoscópicas ${ }^{(12)}$, a investigação eletrorretinográfica é de grande valor diagnóstico na distrofia de cones.

Este estudo ressalta a importância do exame eletrorretinográfico para o diagnóstico precoce confiável e de acompanhamento da evolução da patologia. Na presença de redução na $\mathrm{AV}$ que não possa ser explicada por alteração fundoscópica e/ ou erro refrativo, principalmente se acompanhada de queixas como dificuldade na visão de cores, dificuldade na visão diurna ou fotofobia deve ser feita a investigação eletrorretinográfica para diagnóstico confiável desta condição.

A B S T RA C T

Purpose: To evaluate visual function by full-field electroretinography (ERG) and visual acuity in patients with cone dystrophy. Methods: 23 (7 male and 16 female) patients with suspicion of cone dystrophy were evaluated at the Clinical Electrophysiology of Vision Laboratory of the Universidade Federal de São Paulo. Inclusion criteria were: clinical history, visual acuity measured by ETDRS or Snellen charts and retinal 
function assessed by full-field electroretinography. The peak-topeak amplitude and b-wave implicit time values were compared with previously published age norms. Results: Visual acuity measurement in the best eye ranged from 0.07 to 3.00 logMAR (HM to 20/25) with mean VA of $0.85 \pm 0.64 \log$ MAR (20/140). Mean peak-to-peak amplitude for scotopic rod-response was $144.29 \pm 80.05 \mu \mathrm{V}$ (normal for age) and mean of implicit time was $92.85 \pm 9.02 \mathrm{~ms}$ (normal for age). Mean peak-to-peak amplitude for single-flash cone response was $5.06 \pm 8.59 \mu \mathrm{V}$ (reduced for age) and mean implicit time was $43.4 \pm 14.4 \mathrm{~ms}$ (reduced for age) for detectable responses; for $30 \mathrm{~Hz}$-flicker mean amplitude was $5.00 \pm 9.00 \mu \mathrm{V}$ (reduced for age) and the mean of implicit time was 34.9 $\pm 10.9 \mathrm{~ms}$ (reduced for age). Conclusions: Mean visual acuity was $0.85 \log$ MAR (20/140) in this group of patients with cone dystrophy. Photophobia was the main complaint, followed by decreased vision and defective colour vision. Ten of $23(10 / 23)$ patients had their final diagnosis only after fullfield electroretinography recording, since the fundus examination was normal.

Keywords: Electroretinography; Cones (retina)/physiology; Colour perception; Visual acuity; Low vision

\section{REFERENCIAS}

1. Sadowski B, Zrenner E. Cone and rod function in cone degenerations. Vision Res 1997;37:2303-14.

2. Sloan LL, Brown DL. Progressive retinal degeneration with selective involvement of the cone mechanism. Am J Ophthalmol 1962;54:629-31.

3. François J, De Rouck A, De Laey JJ. Progressive cone dystrophies. Ophthalmologica 1976;173:81-101.

4. Mendonça RHF, Takahashi WY, Usuba FS, Mendonça CHFM. Alterações eletrorretinográficas na distrofia de cones. Rev Bras Oftalmol 1999;58:21-5.

5. Pokorny J, Smith VC, Verriest G, Pinckers AJLG. Congenital and acquired colour vision defects. New York: Grune \& Stratton; 1979.

6. Marmor MF, Zrenner E. Standard for clinical electroretinography (1999 update). International Society for clinical Electrophysiology of Vision. Doc Ophthalmol 1999;97:143-56.

7. Birch DG, Anderson J. Standardized full-field electroretinography. Normal values and their variation with age. Arch Ophthalmol 1992;110:1571-6.

8. Goodman G, Ripps H, Irwin MS. Cone dysfunction syndromes. Arch Ophthalmol 1963;70:126-31.

9. Holopigian K, Seiple W, Greenstein VC, Hood DC, Carr RE. Local cone and rod system function in progressive cone dystrophy. Invest Ophthalmol Vis Sci 2002;43:2364-73.

10. Reichel E, Bruce AM, Sandberg MA, Berson EL. An electroretinographic and molecular genetic study of $\mathrm{x}$-linked cone degeneration. Am J Ophthalmol 1989;18:540-7.

11. Ripps H, Noble KG, Greenstein VC, Siegel IM, Carr RE. Progressive cone dystrophy. Ophthalmology 1987;94:1401-9.

12. Birch DG. Clinical electroretinography. Ophthalmol Clin North Am 1989; 2:469-97.

\section{A B O E LETRÔN I C O}

A versão eletrônica dos Arquivos Brasileiros de 0 ftalmologia com textos completos está disponível em:

- ABO - Arquivos Brasileiros de O ftalmologia

http:/ / www.abonet.com.br

- SCIELO - Scientific Electronic Library O nline

http:/ / www.scielo.org

\section{- Free Medical Journals - http:/ www.freemedicaljournals.com}

